Clinical Vistas

\section{A teenager with fever, rash}

\section{and arthritis}

A I4-year-old boy presented after 5 days of fever, painful polyarthritis in his shoulders, wrists, knees and ankles, and a rash over both lower limbs. He had been caring for 2 pet rats for 3 weeks before his admission but had not been bitten by them.

Fever, a petechial-purpuric rash (Fig. I) with rare pustules on both lower limbs and asymmetric limited polyarthritis were noted on physical examination. Given the exposure history, rat bite fever was considered in the differential diagnosis. Pustular material was cultured in broth supplemented with serum. The suspected bacterium, Streptobacillus moniliformis, was isolated (Fig. 2 and Fig. 3). Notably, biopsy of the petechial rash showed leukocytoclastic vasculitis.

The patient had complete resolution of his symptoms after a 2-week course of oral penicillin and naproxen therapy.

Rat bite fever is a systemic zoonotic illness occurring following exposure to rats or, occasionally, other rodents that o have oropharyngeal colonization with the causative organisms. ${ }^{1}$ In North America, cases are generally due to $S$. moniliformis, whereas Spirillum minus causes cases in Asia.

The name "rat bite fever" is a misnomer, since a bite is not required and

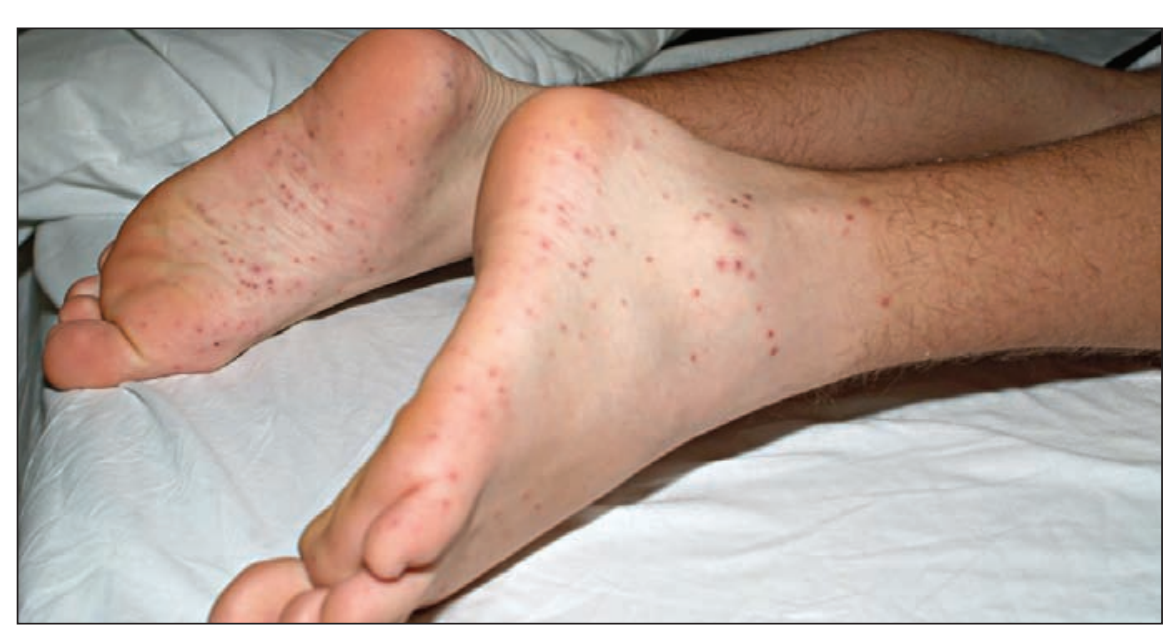

Fig. 1: Petechial-purpuric rash. Biopsy revealed leukocytoclastic vasculitis.

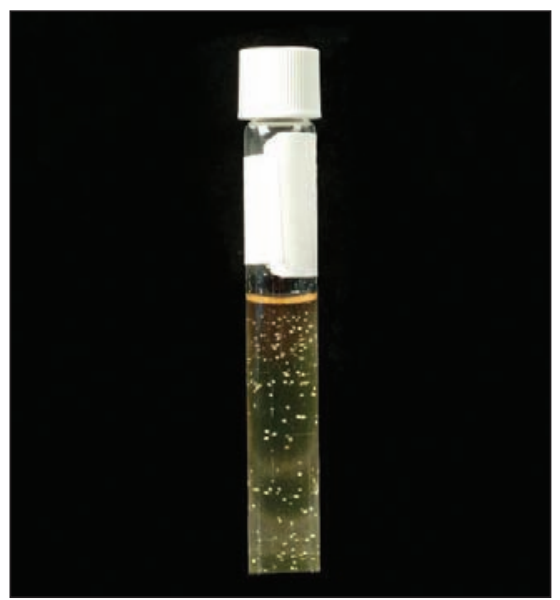

Fig. 2: Characteristic "puff-ball" appearance of Streptobacillus moniliformis in broth culture.

fever may have resolved by the time the patient seeks medical attention. In cases lacking bites, such as ours, there is typically a history of close contact with the oral flora of pet rats, through kissing and sharing food, for example.

The clinical characteristics include fever, rash, myalgias, and arthralgias and arthritis, which occur Io days after exposure. Erythematous macular or papular rashes are typical, but pustules, desquamation and purpuric lesions have been described. Although a case of suspected systemic vasculitis has been reported in association with the condition, ${ }^{2}$ it was not proven histologically, unlike in our case. Disease pathogenesis appears to be due to both direct infectious and immune-mediated mechanisms, as seen here with both culture-

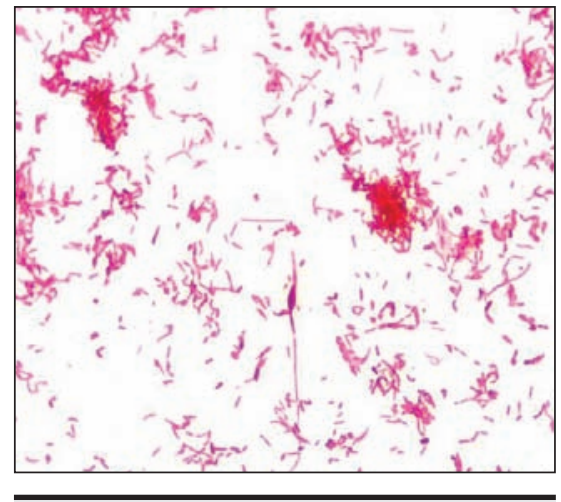

Fig. 3: Unusual Gram stain morphology of Streptobacillus moniliformis: pleomorphic gram-negative bacilli with areas of swelling.

positive and vasculitic skin lesions. The condition is rarely fatal. ${ }^{1}$ Penicillin is the treatment of choice, with intravenous therapy in severe cases.

Our case demonstrates that rat bite fever can occur in Canada following contact with pet rats, even when no bite has occurred. Without a bite history, the diagnosis may be overlooked. Rat bite fever has rarely been reported in Cana$\mathrm{da},{ }^{3}$ but pet rats are now common here. Whether this will result in an increased number of cases is unknown. Presented with an illness consistent with rat bite fever, clinicians should enquire about rodent exposure for a prompt diagnosis and appropriate treatment.

\section{Saif Albedwawi}

Division of Infectious Diseases

The Ottawa Hospital

Claire LeBlanc

Division of Rheumatology

Alyson Show

Department of Pediatrics

Robert William Slinger

Department of Laboratory Medicine

and Pathology

Children's Hospital of Eastern Ontario Ottawa, Ont.

Competing interests: None declared.

\section{REFERENCES}

I. Graves MH, Janda JM. Rat-bite fever (Streptobacillus moniliformis): a potential emerging disease. Int J Infect Dis 200I;5:15I-4.

2. Tattersall RS, Bourne JT. Systemic vasculitis following an unreported rate bite. Ann Rheum Dis 2003;62:605-6.

3. Holden FA, MacKay JC. Rat-bite fever: an occupational hazard. CMAJ I964;9I:78-8I. 